\section{Differential DNA affinity specifies roles for the origin recognition complex in budding yeast heterochromatin}

\author{
Madeleine A. Palacios DeBeer, Ulrika Müller, \\ and Catherine A. Fox ${ }^{1}$
}

Department of Biomolecular Chemistry, University of Wisconsin Medical School, Madison, Wisconsin 53706, USA

The origin recognition complex (ORC) marks chromosomal positions as replication origins and is essential for replication initiation. At a few loci, the ORC functions in heterochromatin formation. We show that the ORC's two roles at the heterochromatic HMRa locus in Saccharomyces cerevisiae were regulated by differences in the ORC's interaction with its target site. At HMRa, a strong ORC-DNA interaction inhibited and delayed replication initiation but promoted heterochromatin formation, whereas a weak ORC-DNA interaction allowed for increased and earlier replication initiation but reduced heterochromatin formation. Therefore, the ORC's interaction with its target site could modulate ORC activity within a heterochromatin domain in vivo.

Supplemental material is available at http://www.genesdev.org.

Received March 24, 2003; revised version accepted June 2, 2003.

The origin recognition complex (ORC) is essential for genome replication in eukaryotes and functions by binding to specific chromosomal positions and recruiting additional proteins essential for origin firing (Bell 2002). Because each chromosome requires the activity of many replication origins for its duplication, ORCs function at hundreds of positions distributed throughout the genome. Although ORCs function at all origins, individual origins vary in activity. Some initiate replication efficiently and early during $S$ phase, whereas others initiate in only a small fraction of cell cycles and later during $S$ phase (Friedman et al. 1997; Yamashita et al. 1997; Polomienko et al. 2001). In budding yeast, a small subset of origins, termed silencers, is associated with the formation of a specialized chromatin structure that represses transcription (Loo and Rine 1995). Significantly, a role for the ORC in repressive chromatin is conserved in metazoans (Pak et al. 1997).

Silencing of HMRa in Saccharomyces cerevisiae is a form of transcription repression requiring a specialized chromatin structure called silent chromatin that is similar to heterochromatin (Pillus and Grunstein 1995; Rusche et al. 2003). Like heterochromatin, silent chromatin causes gene-independent, position-dependent

[Keywords: Heterochromatin; silencers; origins; ORC; yeast] ${ }^{1}$ Corresponding author.

E-MAIL cfox@facstaff.wisc.edu; FAX (608) 262-5253.

Article and publication are at http://www.genesdev.org/cgi/doi/10.1101/ gad.1096703. transcription repression. Both heterochromatin and silent chromatin contain relatively hypoacetylated nucleosomes as well as specialized nonhistone chromatinbinding proteins that help assemble a repressive chromatin domain encompassing many kilobase pairs of DNA. In addition, silent chromatin, like many forms of heterochromatin, is replicated late during $S$ phase (Raghuraman et al. 2001).

DNA elements bound by sequence-specific DNAbinding proteins target specialized chromatin structures to specific chromosomal domains. At $H M R \boldsymbol{a}$, the critical DNA element is the 150-bp $H M R$-E silencer that contains a binding site for the ORC (A-element) as well as a single binding site for each of the abundant nuclear proteins, Raplp and Abflp (Loo and Rine 1995). Together, silencer-binding proteins nucleate the assembly of silenced chromatin by direct physical interactions with nonhistone chromatin-binding proteins called Sirs, which, in turn, recruit additional Sir proteins that modify and bind nucleosomes, forming silent chromatin (Gasser and Cockell 2001).

In addition to its silencer function, the activity of $H M R$-E differs from that of a typical nonsilencer origin such as ARS1 in other ways. $H M R$-E is an inefficient origin, initiating replication in $<10 \%$ of cell cycles (DeBeer and Fox 1999; Polomienko et al. 2001) compared with many nonsilencer origins that initiate replication in a majority of cell cycles (Friedman et al. 1997; Polomienko et al. 2001). Here, we show that the ORC bound the $H M R$-E silencer tightly and that this ORC/silencer interaction enhanced silencer activity but inhibited origin activity of $H M R$-E independently of the chromatin state at HMR $\boldsymbol{a}$.

\section{Results and Discussion}

To compare the relative strengths of the ORC-DNA interaction at $H M R$-E and nonsilencer origins, we performed electrophoretic mobility shift assays (EMSAs) with DNA fragments from several identified origins (ARS elements) within the yeast genome (Fig. 1; Supplementary Table 1). We used ARS1, a well-characterized origin in terms of ORC binding in vitro and origin activity in vivo to guide these experiments (Fig. 1A). ARS1 consists of a conserved 11-bp A-element found in virtually all yeast origins and a less conserved series of $\mathrm{B}$ elements $5^{\prime}$ to the A-rich strand of the A-element (Marahrens and Stillman 1992). The A-element is essential for replication initiation in vivo and ORC binding in vitro (Bell and Stillman 1992; Marahrens and Stillman 1992). The B-elements contribute to but are not essential for replication initiation. Mutations in the B1-element reduce ORC binding in vitro, and footprinting and crosslinking experiments indicate that the ORC contacts a bipartite element in ARS1 consisting of the B1- and Aelements (B1/A-element; Rao and Stillman 1995; Lee and Bell 1997).

Other origins that have been examined contain A- and $\mathrm{B}$-elements including a B1-element in similar relative positions as those in ARS1 (Fig. 1B, see ARS305; Huang and Kowalski 1996). Also, a sequence comparison of multiple origins revealed an expanded ORC consensusbinding site (Fig. 1B) that includes both the A-element and sequences expected to contain a B1-element (Theis 
Palacios DeBeer et al.

Figure 1. ORC binding to the B1/A-elements of various origins. $(A)$ Structural organization of $A R S 1$ as defined by linker-scanning mutagenesis (Marahrens and Stillman 1992) and the HMR-E silencer as defined by deletion analysis (for review, see Loo and Rine 1995). A B1-element (?) for $H M R$-E was functionally characterized in this study. (B) Sequences of B1/A-elements for a variety of origins examined for ORC binding in vitro. B1-elements as defined by mutagenesis are shown for ARS1 and ARS305 with boxes above the element (Marahrens and Stillman 1992; Huang and Kowalski 1996). The "expanded" ORC consensus site is shown at the bottom (Theis and Newlon 1997). The conserved A-element is shown in larger, bold font and underlined. The conserved nucleotides in the more variable B1-element are shown as bold and underlined. The * marks B1/Aelements in which every nucleotide in the fragment other than the conserved nucleotides of the expanded ORC consensus site has been changed to $\mathrm{G}$ or $\mathrm{C}$ (GC-E) or A and $\mathrm{T}$ (A/T-E). (C) Equilibrium binding of purified ORC to B1/A-elements of indicated origins as measured by EMSAs (Bell et al. 1995; Rao and Stillman 1995). Binding reactions used $0.1 \mathrm{nM}$ radiolabeled DNA and $0 \mathrm{nM}$ (lanes 1,6,11,16), $0.1 \mathrm{nM}$ (lanes 2,7,12,17), $1 \mathrm{nM}$ (lanes $3,8,13,18$ ), and $10 \mathrm{nM}$ (lanes $4,5,9,10,14,15,19,20)$ ORC. All binding reactions contained $2 \mathrm{mM}$ ATP except those in lanes marked NA (No ATP; lanes 5,10,15,20). $(D)$ Competition binding experiments to measure relative affinities of ORC for HMR-E and ARS1. An EMSA was performed with 0.02 nM radiolabeled $H M R$-E DNA (as in $B$ ) and ORC to obtain $~ 50 \%$ binding of DNA substrate (lane 1). The ORC/HME-E complex is indicated by an *. Increasing concentrations of cold $H M R$-E substrate DNA (lanes 2-5) or ARS1 substrate DNA (as in B; lanes 6-9) were added to identical reactions to determine the concentrations needed to compete the binding of ORC to radiolabeled HMR-E. (Lane 10) A no-ATP control experiment. The concentrations of cold DNA substrate were $0.6 \mathrm{nM}$ (lanes 2,6), $1 \mathrm{nM}$ (lanes 3,7), $2 \mathrm{nM}$ (lanes 4,8), and $20 \mathrm{nM}$ (lanes 5,9). (E) ORC binding as described in $C$ to the natural (HMR-E) and synthetic silencer's (HMR-SS) putative B1/Aelements and B1/A hybrid DNA templates as shown in $B$.

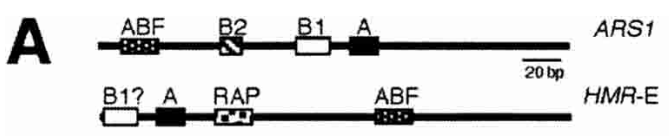

B ARS1 1 B1 HMA-E TTTTAMATCGAATTTANTACCTAAATATAAAAAATGTTATTAT ARS305 ARS607 HMRSS SS-E E-SS *G/C-E "AT-E ARS605 TTGTAAAGTTTC CI TTAGCAACAAAACGTAATTAATTCTTTGGT

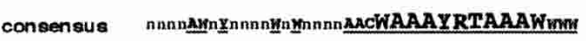

C

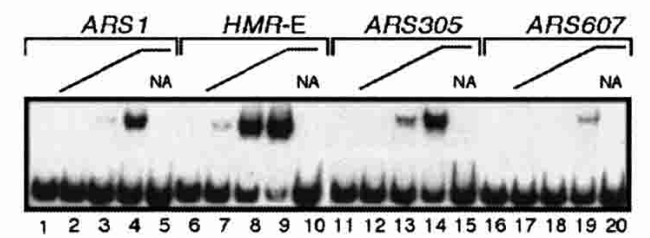

D
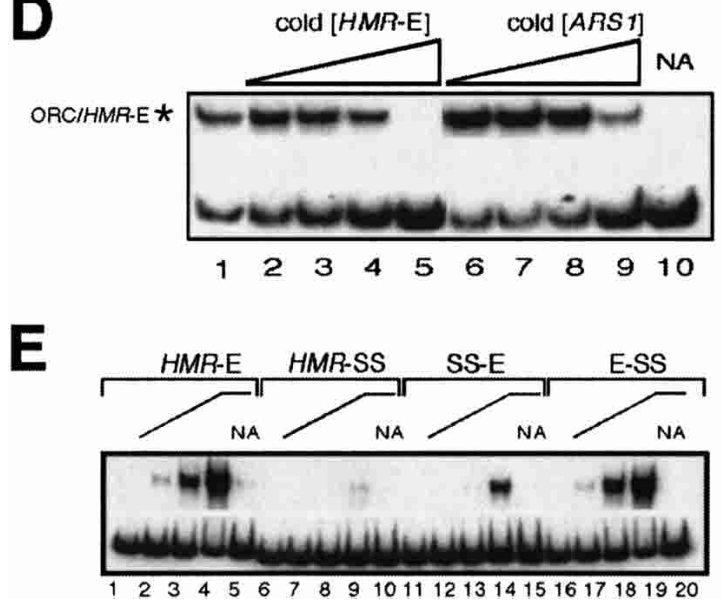

tributed to high-affinity binding by ORC, we used an engineered version of HMR-E called the synthetic silencer (HMR-SS) whose replication and silencing activities have been well defined (McNally and Rine 1991; Fox et al. 1995). HMR-SS contains an A-element and binding sites for Raplp and Abflp positioned with the same spacing and order as in $H M R$-E. However, the binding sites as well as the sequences between them differ between $H M R$-E and $H M R$-SS. We noted that $H M R$-SS contains a poor match to the expanded ORC consensus site, particularly in the region that should contain a B1-element (Fig. 1B). Therefore, we tested whether the putative B1/ A-element of $H M R$-SS would bind ORC with a lower affinity compared with $H M R$-E. Significantly, HMR-SS bound ORC with $>100$-fold lower affinity than HMR-E (Fig. 1E), even though HMR-SS initiates replication more efficiently than $H M R$-E on the chromosome (Fox et al. 1995; DeBeer and Fox 1999). 
To test whether the B1-element contributed to highaffinity ORC binding at $H M R-\mathrm{E}$, we engineered hybrid B1/A-elements from $H M R$-SS and $H M R$-E and tested them in EMSAs (Fig. 1B,E). A hybrid DNA element containing the B1-element from $H M R$-SS and the A-element from HMR-E (SS-E) bound ORC only slightly better than the B1/A-element from HMR-SS. However, the hybrid element containing the B1-element of $H M R$-E and the A-element of HMR-SS (E-SS) bound ORC as tightly as the B1/A-element of $H M R$-E (Fig. 1E). Therefore, $H M R$-E contains a B1-element important for high-affinity binding by ORC.

We next tested whether a high-affinity ORC-DNA interaction within a silencer could contribute to the silencer's origin or silencing activities at HMRa. As described above, $H M R-E$ is an extremely weak replication origin but a strong silencer. In contrast, $H M R$-SS is a stronger origin but a weaker silencer, and both of its activities are reduced by the orc2-1 mutation (Fox et al. 1995; DeBeer and Fox 1999). Therefore, we engineered two strains that differed only in terms of the silencer that controlled their HMR $\boldsymbol{a}$ locus (Fig. 2A). One strain contained $H M R$-SS as the only silencer at HMR $\boldsymbol{a}$ (lowaffinity silencer). The other strain contained a hybrid version of $H M R$-E and $H M R$-SS in which the low-affinity ORC-binding site (B1/A-element) of HMR-SS was replaced with the high-affinity ORC-binding site of HMR-E (high-affinity silencer; Fig. 2A). These strains were examined for replication (Figs. 2B, 3) and silencing (Fig. 4) of HMR $\boldsymbol{a}$.

Significantly, at $H M R \boldsymbol{a}$, the low-affinity silencer initiated replication more efficiently than the high-affinity silencer as determined by $2 \mathrm{D}$ origin mapping gels (Fig. 2B; SIR2), indicating that the high-affinity ORC-binding site could inhibit origin activity at HMR $\boldsymbol{a}$.

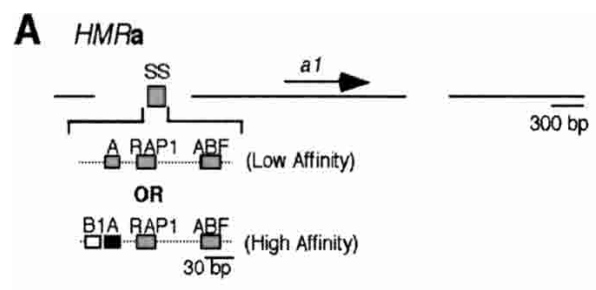

B

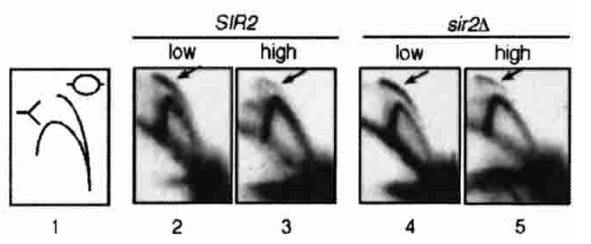

Figure 2. A high-affinity ORC-binding site inhibits replication initiation by the HMR-SS silencer. (A) Structure of the two versions of $H M R \boldsymbol{a}$ analyzed for origin function in vivo. The $H M R$-I silencer and the cryptic origins flanking the defined $H M R$-E silencer were deleted (shown as gaps; DeBeer and Fox 1999). In one strain, the HMR-SS containing a low-affinity ORC-binding site was the only silencer at $H M R \boldsymbol{a}$ (low affinity). In a second strain, an HMR-SS engineered to contain the high-affinity ORC-binding site of natural $H M R$-E was the only silencer at HMRa (high affinity). (B) 2D origin analysis of the HMR-SS origins described in A in both SIR2 and sir2A strains. $2 \mathrm{D}$ origin mapping experiments allow the analysis of chromosomal replication intermediates formed in vivo by allowing the separation of origin bubbles from replication forks (Friedman and Brewer 1995). Arrows indicate origin bubbles. Except for differences noted, the strains were MAT $\alpha$ and isogenic to W303.
In some contexts, SIR-dependent silent chromatin can inhibit replication initiation and cause early origins to fire later in S phase (Stevenson and Gottschling 1999; Zappulla et al. 2002). However, HMR $\boldsymbol{a}$ is replicated late in $\mathrm{S}$ phase even in strains containing SIR gene mutations that eliminate SIR-dependent silent chromatin (Dubey et al. 1991). In addition, the low efficiency of replication initiation at HMR $\boldsymbol{a}$ is not affected dramatically by deletions in SIR genes (Rivier and Rine 1992; Fox et al. 1995; DeBeer and Fox 1999). Thus, some feature other than the heterochromatic state inhibits replication initiation in this region. Therefore, we tested whether a high-affinity ORC/DNA interaction within a silencer could inhibit replication initiation by $H M R$-E in cells containing a deletion of the SIR2 gene (Fig. 2B). Significantly, the highaffinity silencer still initiated replication less efficiently than the low-affinity silencer as determined by the reduced number of bubble intermediates observed in 2D origin mapping experiments (Fig. 2B; sir2 $\Delta$ ). Thus, the SIR-dependent chromatin state could not explain the inhibited origin firing at $H M R \boldsymbol{a}$.

Silent chromatin, like many forms of heterochromatin, is one of the last regions of the genome to be replicated. To test whether inhibiting replication initiation at $H M R \boldsymbol{a}$ via a strong ORC-binding site contributed to the late replication time of this locus, we compared replication timing of $H M R \boldsymbol{a}$ and two other well-characterized chromosomal regions, $A R S 1$ and R11, during $\mathrm{S}$ phase in strains that differed only in terms of their silencers (Fig. 2A).

2D origin mapping performed on samples harvested during $S$ phase in cell cycle arrest and release experiments revealed that a strong silencer ORC-binding site caused a later replication timing profile of $H M R \boldsymbol{a}$ (Fig. 3). $H M R \boldsymbol{a}$ containing the low-affinity silencer showed measurable replication intermediates, including origin bubbles as early as 40 min after release into S phase (Fig. $3 \mathrm{~A})$. In contrast, $H M R \boldsymbol{a}$ containing the high-affinity silencer did not show replication intermediates until 50 min, and origin bubbles were first and barely detectable at $70 \mathrm{~min}$ (Fig. 3A). The replication-timing profiles of the late $(R 11)$ and mid-early $(A R S 1)$ controls were similar in the two strains (Fig. 3B). Thus, the low-affinity silencer led to substantial replication of $H M R \boldsymbol{a}$ prior to the peak replication time of $R 11$ (40-60 min for HMR $\boldsymbol{a}$ vs. 50-60 min for R11), whereas the high-affinity silencer led to a replication peak for $H M R \boldsymbol{a}$ that occurred after the peak replication time for $R 11$ (70 $\mathrm{min}$ for $H M R \boldsymbol{a}$ vs. $60 \mathrm{~min}$ for R11). To the best of our knowledge, this is the first example of any mutation affecting the replication time of HMR a.

Because SIR-dependent silent chromatin can affect the time during $S$ phase at which an origin fires (Stevenson and Gottschling 1999; Zappulla et al. 2002), we tested whether it had an effect on the relative replication-timing profiles of the low- and high-affinity silencers at HMR a (Fig. 3C,D). Significantly, the SIR2 genotype had a negligible effect on the absolute or the relative replication timing profiles of HMR $\boldsymbol{a}$ compared with R11 and ARS1. Thus, a silencer that can bind ORC tightly inhibits replication origin activity at HMR $\boldsymbol{a}$ and contributes to the SIR-independent late replication time of this locus.

We also tested whether a strong ORC-binding site was important for silencing of HMR $\boldsymbol{a}$. We examined relevant yeast strains with silencers as described above (Fig. 2A) 
Palacios DeBeer et al.

A

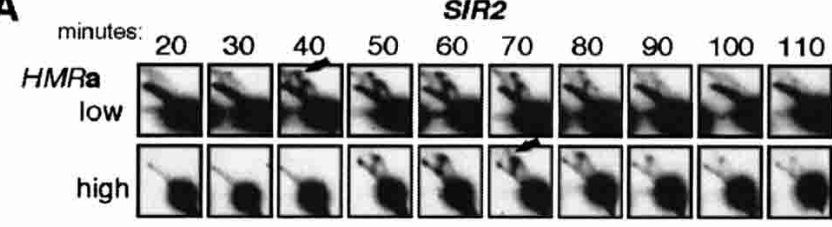

C

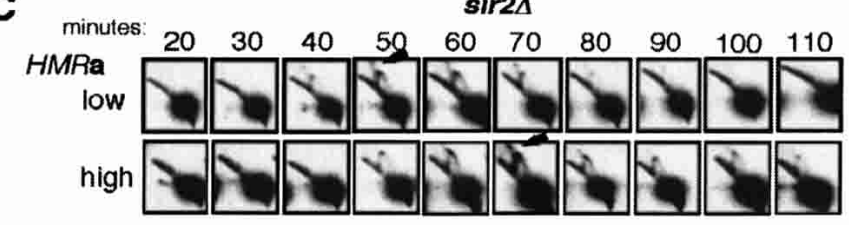

B

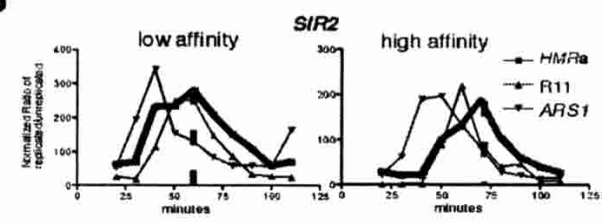

D

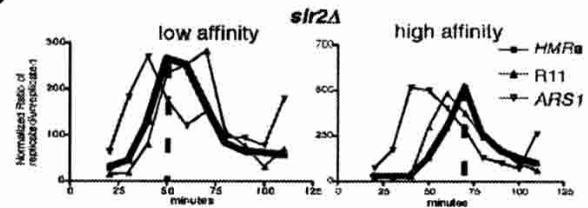

Figure 3. A high-affinity ORC-binding site in $H M R$-SS delayed the replication time of $H M R \boldsymbol{a}$. $(A)$ Two strains, differing only in terms of their silencers at HMR a (Fig. 2A) were examined by 2D origin analysis at 10-min intervals throughout $\mathrm{S}$ phase after release from synchronized G1 arrest. Arrows indicate the first detectable origin bubbles. (B) A plot of quantified data obtained from analysis of replication intermediates for $H M R, R 11$, and ARS1 for the two strains examined in A. (C) Replication times of HMR $\boldsymbol{a}$ controlled by the two different versions of HMR-SS were not affected by SIR genotype. The same experiments as in $A$ were performed in sir2 $\Delta$ strains. Arrows indicate the peaks of replication. $(D)$ A plot of quantified data obtained from analysis of replication intermediates for $H M R, R 11$, and $A R S 1$ for strains examined in $C$. For $B$ and $D$, replication data for $H M R, R 11$, and $A R S 1$ were graphed as ratios of replication intermediates to unreplicated linear DNA ( $Y$-axis) versus time after release from G1 arrest ( $X$-axis). Data were quantified using a PhosphorImager. The peak replication time for HMR $\boldsymbol{a}$ in each strain is indicated with a broken vertical line.

for their ability to silence $H M R \boldsymbol{a}$ (Fig. 4A). HMR-SS (lowaffinity silencer) is a weak silencer that provides for only partial silencing of HMR a (Fox et al. 1995). This reduced ability to form silent chromatin can be measured by the appearance of a1 mRNA transcribed from HMR $\boldsymbol{a}$ in an RNA blot hybridization experiment (Fig. 4A, lane 1). The orc2-1 mutation completely abolished the low-affinity silencer's ability to silence (Fig. 4A, lane 2). In contrast, the high-affinity silencer provided for full silencing of $H M R \boldsymbol{a}$ (Fig. 4A, lanes 3,10 ) that was unaffected by the orc2-1 mutation (Fig. 4A, lane 4). Thus, the high-affinity ORC-binding site, owing in large part to the B1-element
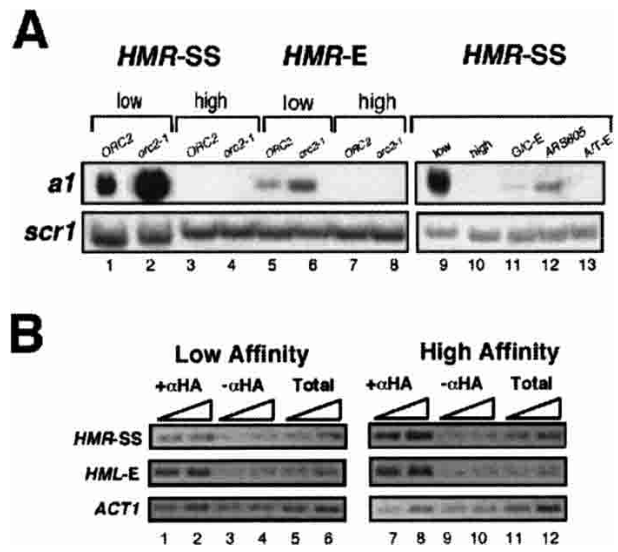

Figure 4. A high-affinity ORC-binding site contributes to silencing of and efficient Sirlp binding to HMR a. (A) RNA blot hybridization of $a 1$ mRNA expressed from $H M R \boldsymbol{a}$ in strains containing the $H M R$ SS (lanes 1-4) or natural HMR-E (lanes 5-8) silencers with the indicated B1/A-element ORC-binding site and either ORC2 (lanes $1,3,5,7)$ or the orc2-1 allele (lanes 2,4,6,8). Hybrid versions of $H M R$ SS containing high-affinity ORC-binding sites different from the B1/A-element of $H M R-E$ (see Fig. 1B) also silenced HMR $\boldsymbol{a}$ (lanes 9-13). scr1 RNA was used as an RNA loading control. (B) ChIP of Sirlp-3xHA in yeast strains with either the low-affinity or highaffinity silencers was performed as described previously (Gardner and Fox 2001) except that $H M L-E$ was also examined as an internal control with $H M L$-specific primers. at $H M R$-E (Fig. 1D), was required for ORC-dependent silent chromatin formation.

These data indicated that a high-affinity ORC-binding site was sufficient to convert the weak $H M R$-SS silencer into a strong silencer whose ability to silence $H M R \boldsymbol{a}$ was insensitive to the orc2-1 mutation. Because the synthetic silencer is an engineered version of $H M R$-E that contains differences in addition to the A/B1-element, we also tested the effect of a low-affinity ORC-binding site on natural HMR-E (Fig. 4A, lanes 5-8). A low-affinity ORC-binding site reduced silencing by $H M R$-E and made it more sensitive to orc2-1 (Fig. 4A, lanes 5,6). The silencer activity of $H M R$-E was not as sensitive to a lowaffinity ORC-binding site as $H M R$-SS, consistent with other differences between the two elements having roles in silencer strength. Nevertheless, these data indicate that a strong ORC-binding site was necessary for full silencing of $H M R \boldsymbol{a}$.

Conceivably, some unique sequence in the B1-element of HMR-E could promote the ORC's function in silencing rather than high-affinity binding per se. To test this, we engineered silencers containing high-affinity B1/Aelements that were relatively "unrelated" to the B1/Aelement of $H M R$-E (Fig. 1B). These hybrid silencers, including one that contained the B1/A-element from the exceptional nonsilencer origin ARS605 that bound ORC with a particularly high affinity in vitro (Supplementary Table 1) silenced HMR $\boldsymbol{a}$ more effectively than the lowaffinity silencer, suggesting that a high-affinity binding site rather than some specific sequence was important for silencer function at HMR a (Fig. 4A, lanes 9-13).

One mechanism to reduce silencing at $H M R \boldsymbol{a}$ is to reduce the recruitment of Sir proteins; however, Sir recruitment alone is not sufficient for silencing $H M R \boldsymbol{a}$ (Lau et al. 2002). Because the primary role for ORC in silencing HMR $\boldsymbol{a}$ is to recruit Sirlp (for review, see Gasser and Cockell 2001), we tested whether the high-affinity ORC-binding site was necessary for efficient Sirlp binding in vivo by chromatin immunoprecipitation /ChIP; Fig. 4B). Significantly, Sirlp-3xHA bound more efficiently to $H M R \boldsymbol{a}$ in the strain controlled by the silencer 
with a high-affinity ORC-binding site, indicating that this site contributes to Sirlp's association with $H M R \boldsymbol{a}$.

A major conclusion of this study is that stable binding of ORC to its target site in HMR $\boldsymbol{a}$ favors ORC's role in silencing over its role in replication initiation at this locus. We propose that the low origin activity at $H M R \boldsymbol{a}$ is not necessarily a prerequisite for silent chromatin formation, but rather a consequence of a stable ORC-chromosome interaction that is optimal for formation of silent chromatin. Tight binding by ORC would enhance the probability of a Sir complex remaining stably associated with $H M R$-E because ORC binds Sirlp (Triolo and Sternglanz 1996), and this, in turn, would stabilize silent chromatin at HMRa. Alternatively, the tight binding may reflect a conformation of the ORC at the silencer optimal for recruitment of Sirlp. The late replication time of $H M R \boldsymbol{a}$ during $S$ phase may therefore be a consequence of an ORC-DNA interaction optimized for Sirlp recruitment rather than replication.

This study also provides an explanation for why defects in ORC caused by mutations in ORC genes fail to cause either silencing or replication defects at wild-type $H M R \boldsymbol{a}$ and why compromised silencers were useful in identifying the first mutant alleles of ORC genes (Foss et al. 1993). For example, the orc2-1 mutant allele lowers the concentration of ORC in vivo (Shimada et al. 2002) and reduces replication initiation by many origins, including the HMR-SS silencer origin (Fox et al. 1995; Liang et al. 1995). However, because the $H M R$-E silencer has a high affinity for ORC, it is resistant to reductions in ORC concentration and may have a competitive advantage over other lower-affinity chromosomal origins in an orc2-1 mutant strain.

The observation that the high-affinity ORC-binding site did not promote enhanced replication initiation at $H M R \boldsymbol{a}$ compared with the low-affinity silencer may seem nonintuitive at one level because such a site should increase the probability that the silencer origin is bound by ORC in any given cell, and ORC binding is necessary for origin activity. However, it is clear that there is enough ORC present in yeast to occupy origins with measurably lower affinities sufficiently enough that they initiate in a majority of cell cycles (Supplementary Table 1). Perhaps the silencer origin used here is revealing something about ORC function in replication initiation that is not possible to observe at nonsilencer origins optimized for origin firing. If true and if the highaffinity binding by ORC to silencer DNA observed in this study reflects a slow off-rate, then perhaps a step in replication initiation requires the ORC's dissociation from double-stranded origin DNA. Such a mechanism would be akin to Escherichia coli RNA polymerase promoter clearance (for review, see Hsu 2002). Alternatively, other features of the silencer origin may collaborate with some as yet unknown feature of the $H M R-\mathrm{E}$ A/B1-element that influences both ORC binding affinity and conformation and Sir binding, resulting in low origin efficiency and late replication of HMR-E.

Interestingly, genome-wide binding studies of ORC and MCM proteins reveal that the heterochromatic loci $H M R \boldsymbol{a}$ and $H M L \alpha$ are the only genomic regions that exhibit inefficient origin activity yet bind both ORC and MCM in vivo quite efficiently as measured by ChIP experiments (Wyrick et al. 2001). These data support the idea that origin inefficiency associated with a stable ORC-chromosome interaction may reflect the role for
ORC in silencing. Our data indicate that at $H M R \boldsymbol{a}$, a significant contributor to a stable ORC-chromosome interaction may be the ORC DNA-binding site (B1/A-element) itself. But it is probable that other features of silencers and heterochromatic regions in yeast and metazoans could contribute substantially to a stable interaction between the ORC and its chromosomal target site.

Silencers use several of the same proteins necessary for both replication initiation and transcription activation at other loci, and a central question is how these proteins can perform different functions depending on their genome position. We propose that highly stable binding of these factors to silencers may be critical for their roles in forming a stable silent chromatin structure but less essential, and possibly even detrimental, for their other roles at other positions. Further biochemical analysis of silencer-protein complexes from yeast and other organisms should address this idea and help define the mechanisms that govern the duplication and expression of the eukaryotic genome.

\section{Materials and methods}

Yeast strains

All experiments were carried out with yeast strains isogenic to W303-1A. Chromosomal gene deletions and mutations were generated using standard recombinant DNA technology and yeast molecular biology.

In vitro ORC-binding assays

The DNA probes for EMSAs were prepared by annealing complementary 55-bpair oligonucleotides containing the core 11-bp ORC-binding site (A-element) and best match to the $5^{\prime}$ putative B1-element from relevant ARSs (Fig. 1B). The annealed fragments were subcloned into pSTBlue 1 (Novagen) and then used as templates to generate 260-bp radiolabeled probes $\left({ }^{32} \mathrm{P}\right.$-dCTP) by PCR with a set of common primers complementary to pSTBlue1. Because all A/B1-elements contained the same flanking sequences, the specific activity of probes was virtually the same, and DNA concentrations of different probes varied by at most 1.2 -fold. The binding reactions were done under equilibrium conditions in which ORC was in excess over probe DNA such that the concentration of ORC necessary to bind $50 \%$ of the probe DNA was approximately equal to the apparent $K_{\mathrm{d}}$. Binding reactions contained $20 \mu \mathrm{g} / \mathrm{mL} \mathrm{dI}-\mathrm{dC}$ as nonspecific competitor (Amersham Pharmacia). The concentrations of ORC and DNA used in reactions are indicated in the figure legends.

$2 D$ origin analysis and replication timing experiments

2D origin analysis was performed as described (Friedman and Brewer 1995; Kim and Huberman 2001). For replication-timing experiments, the strains were as described in Figure 2 except they were additionally engineered to contain MATa and bar1D::HIS3 for arrest and release experiments. To examine the effect that silent chromatin had on replicationtiming profiles of $H M R \boldsymbol{a}$, the strains were additionally modified to contain sir2 $\Delta:: U R A 3$ and $h m l \Delta:: L E U 2$. First, $4 \mathrm{~L}$ of yeast cells was grown in YPD to 0.7 O.D. and concentrated for arrest in G1 with $\alpha$-factor at $23^{\circ} \mathrm{C}$. Arrested cells were harvested, washed, and released into S phase in YPD plus $0.025 \mathrm{mg} / \mathrm{mL}$ Pronase (CalBiochem). Every $10 \mathrm{~min}, 400 \mathrm{~mL}$ of cells was harvested and genomic DNA was isolated for each sample. The DNA was split into three samples and analyzed for relevant replication intermediates directly after restriction digest. To examine replication intermediates, genomic DNA was digested with HindIII (for $H M R \boldsymbol{a}$ ), with NcoI (for ARS1), and with EcoRI (for R11). Hybridization probes were generated by PCR with appropriate primers.

\section{Acknowledgments}

We thank Bruce Stillman for the ORC baculoviruses and purification protocol; Michael Weinreich for additional protocols; Jim Dahlberg for critical reading of the manuscript; and Paul Kaufman, Ann Kirchmaier, and Michael Sheets for productive discussions. We also thank J.A. Huber- 
man and M.K. Raghuraman for technical advice on replication timing experiments. This work was supported by predoctoral fellowships from the NSF and Ford Foundation (to M.A.P.D.), and by a grant from the NIH (GM056890 to C.A.F).

The publication costs of this article were defrayed in part by payment of page charges. This article must therefore be hereby marked "advertisement" in accordance with 18 USC section 1734 solely to indicate this fact.

\section{References}

Bell, S.P. 2002. The origin recognition complex: From simple origins to complex functions. Genes \& Dev. 16: 659-672.

Bell, S.P. and Stillman, B. 1992. ATP-dependent recognition of eukaryotic origins of DNA replication by a multiprotein complex. Nature 357: $128-134$

Bell, S.P., Mitchell, J., Leber, J., Kobayashi, R., and Stillman, B. 1995. The multidomain structure of Orclp reveals similarity to regulators of DNA replication and transcriptional silencing. Cell 83: 563-568.

DeBeer, M.A.P. and Fox, C.A. 1999. A role for a replicator dominance mechanism in silencing. EMBO J. 18: 3808-3819.

Dubey, D.D., Davis, L.R., Greenfeder, S.A., Ong, L.Y., Zhu, J.G., Broach, J.R., Newlon, C.S., and Huberman, J.A. 1991. Evidence suggesting that the ARS elements associated with silencers of the yeast matingtype locus $H M L$ do not function as chromosomal DNA replication origins. Mol. Cell. Biol. 11: 5346-5355.

Foss, M., McNally, F.J., Laurenson, P., and Rine, J. 1993. Origin recognition complex (ORC) in transcriptional silencing and DNA replication in S. cerevisiae. Science 262: 1838-1844.

Fox, C.A., Loo, S., Dillin, A., and Rine, J. 1995. The origin recognition complex has essential functions in transcriptional silencing and chromosomal replication. Genes \& Dev. 9: 911-924.

Friedman, K.L. and Brewer, B.J. 1995. Analysis of replication intermediates by two-dimensional agarose gel electrophoresis. Methods Enzymol. 262: 613-627.

Friedman, K.L., Brewer, B.J., and Fangman, W.L. 1997. Replication profile of Saccharomyces cerevisiae chromosome VI. Genes to Cells 2: 667678.

Gardner, K.A. and Fox, C.A. 2001. The Sir1 protein's association with a silenced chromosome domain. Genes \& Dev. 15: 147-157.

Gasser, S.M. and Cockell, M.M. 2001. The molecular biology of the SIR proteins. Gene 279: 1-16.

Hsu, L.M. 2002. Promoter clearance and escape in prokaryotes. Biochim. Biophys. Acta 1577: 191-207.

Huang, R.Y. and Kowalski, D. 1996. Multiple DNA elements in ARS305 determine replication origin activity in a yeast chromosome. Nucleic Acids Res. 24: 816-823.

Kim, S.M. and Huberman, J.A. 2001. Regulation of replication timing in fission yeast. $E M B O$ J. 20: 6115-6126.

Lau, A., Blitzblau, H., and Bell, S.P. 2002. Cell cycle control of the establishment of mating-type silencing in S. cerevisiae. Genes \& Dev. 16: 2935-2945.

Lee, D.G. and Bell, S.P. 1997. Architecture of the yeast origin recognition complex bound to origins of DNA replication. Mol. Cell. Biol. 17: 7159-7168.

Liang, C., Weinreich, M., and Stillman, B. 1995. ORC and Cdc6p interact and determine the frequency of initiation of DNA replication in the genome. Cell 81: 667-676.

Loo, S. and Rine, J. 1995. Silencing and domains of heritable gene expression. Annu. Rev. Cell Dev. Biol. 11: 519-548.

Marahrens, Y. and Stillman, B. 1992. A yeast chromosomal origin of DNA replication defined by multiple functional elements. Science 255: 817-823.

McNally, F.J. and Rine, J. 1991. A synthetic silencer mediates SIR-dependent functions in Saccharomyces cerevisiae. Mol. Cell. Biol. 11: 5648-5659.

Pak, D.T., Pflumm, M., Chesnokov, I., Huang, D.W., Kellum, R., Marr, J., Romanowski, P., and Botchan, M.R. 1997. Association of the origin recognition complex with heterochromatin and HP1 in higher eukaryotes. Cell 91: 311-323.

Pillus, L. and Grunstein, M. 1995. Chromatin structure in epigenetic regulation in yeast. In Chromatin structure and gene expression (ed. S.C.R. Elgin), pp. 123-141. Oxford University Press, New York.
Polomienko, A., Dershowitz, A., De, J., and Newlon, C.S. 2001. Completion of replication map of Saccharomyces cerevisiae chromosome III. Mol. Cell. Biol. 12: 3317-3327.

Raghuraman, M.K., Winzeler, E.A., Collingwood, D., Hunt, S., Wodicka, L., Conway, A., Lockhart, D.J., Davis, R.W., Brewer, B.J., and Fangman W.L. 2001. Replication dynamics of the yeast genome. Science 294: 115-121.

Rao, H. and Stillman, B. 1995. The origin recognition complex interacts with a bipartite DNA binding site within yeast replicators. Proc. Nat1. Acad. Sci. 92: 2224-2228.

Rivier, D.H. and Rine, J. 1992. An origin of DNA replication and a transcriptional silencer require a common element. Science 256: 659663.

Rusche, L.N., Kirchmaier, A.L., and Rine, J. 2003. The establishment, inheritance, and function of silenced chromatin in Saccharomyces cerevisiae. Annu. Rev. Biochem. 72: 481-516.

Shimada, K., Pasero, P., and Gasser, S.M. 2002. ORC and the intra-Sphase checkpoint: A threshold regulates Rad53p activation in $\mathrm{S}$ phase. Genes \& Dev. 16: 3236-3252.

Stevenson, J.B. and Gottschling, D.E. 1999. Telomeric chromatin modulates replication timing near chromosome ends. Genes \& Dev. 13: 146-151.

Theis, J.F. and Newlon, C.S. 1997. The ARS309 chromosomal replicator of Saccharomyces cerevisiae depends on an exceptional ARS consensus sequence. Proc. Nat1. Acad. Sci. 94: 10786-10791.

Triolo, T. and Sternglanz, R. 1996. Role of interactions between the origin recognition complex and SIRl in transcriptional silencing. $\mathrm{Na}$ ture 381: 251-253.

Wyrick, J.J., Aparicio, J.G., Chen, T., Barnett, J.D., Jennings, E.G., Young, R.A., Bell, S.P., and Aparicio, O.M. 2001. Genome-wide distribution of ORC and MCM proteins in S. cerevisiae: High-resolution mapping of replication origins. Science 14: 2357-2360.

Yamashita, M., Hori, Y., Shinomiya, T., Obuse, C., Tsurimoto, T., Yoshikawa, H., and Shirahige, K. 1997. The efficiency and timing of initiation of replication of multiple replicons of Saccharomyces cerevisiae chromosome VI. Genes to Cells 2: 655-665.

Zappulla, D.C., Sternglanz, R., and Leatherwood, J. 2002. Control of replication timing by a transcriptional silencer. Curr. Biol. 12: 869-875. 


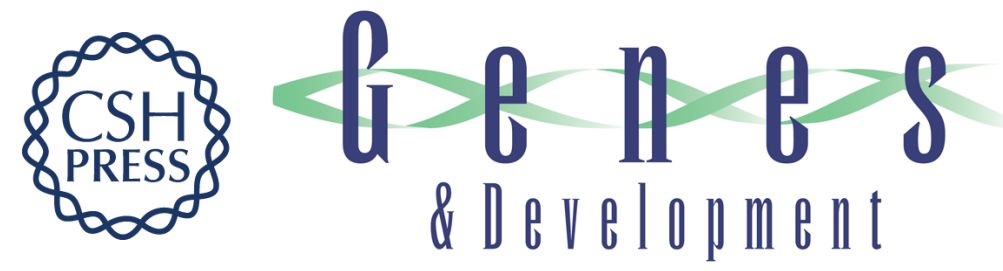

\section{Differential DNA affinity specifies roles for the origin recognition complex in budding yeast heterochromatin}

Madeleine A. Palacios DeBeer, Ulrika Müller and Catherine A. Fox

Genes Dev. 2003, 17:

Access the most recent version at doi:10.1101/gad.1096703

Supplemental http://genesdev.cshlp.org/content/suppl/2003/07/08/17.15.1817.DC1
Material

References This article cites 33 articles, 16 of which can be accessed free at:

http://genesdev.cshlp.org/content/17/15/1817.full.html\#ref-list-1

License

Email Alerting

Receive free email alerts when new articles cite this article - sign up in the box at the top

Service

right corner of the article or click here.

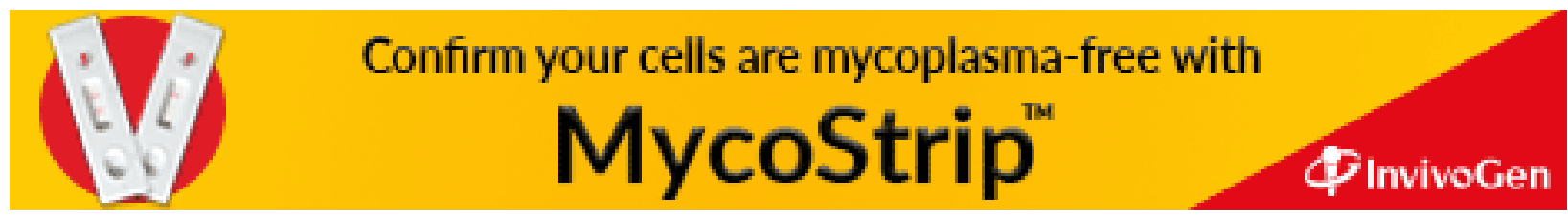

